\title{
Tough, bio-inspired hybrid materials
}

\author{
E. Munch, ${ }^{1}$ M. E. Launey, ${ }^{1}$ D. H. Alsem, ${ }^{1,2}$ E. Saiz, ${ }^{1}$ A.P. Tomsia, ${ }^{1}$ R. O. Ritchie $^{1,3 *}$
}

The notion of mimicking natural structures in the synthesis of new structural materials has generated enormous interest but has yielded few practical advances. Natural composites achieve strength and toughness through complex hierarchical designs extremely difficult to replicate synthetically. Here we emulate Nature's toughening mechanisms through the combination of two ordinary compounds, aluminum oxide and polymethylmethacrylate, into ice-templated structures whose toughness can be over 300 times (in energy terms) that of their constituents. The final product is a bulk hybrid ceramic material whose high yield strength and fracture toughness ( 200 MPa and $\sim 30$ $\mathrm{MPa} \sqrt{ } \mathbf{m})$ provide specific properties comparable to aluminum alloys. These model materials can be used to identify the key microstructural features that should guide the synthesis of bio-inspired ceramic-based composites with unique strength and toughness.

With the quest for more efficient energy-related technologies, there is an imperative to develop lightweight, high-performance structural materials that possess both exceptional strength and toughness. Unfortunately, these two properties tend to be mutually exclusive and the attainment of optimal mechanical performance is invariably a compromise often achieved through the empirical design of microstructures. Nature has long developed the ability to combine brittle minerals and organic molecules into hybrid composites with exceptional fracture resistance and structural capabilities (1-3); indeed, many natural materials like bone, wood and nacre (abalone shell) have highly sophisticated structures with complex hierarchical designs whose properties are far in excess what could be expected from a simple mixture of their components $(2,4)$. Biological mineralized composites, in particular bone, dentin and nacre (5-7), can generate fracture toughness (i.e., resistance to the initiation and growth of a crack) primarily by extrinsic toughening mechanisms (8) that "shield" any crack from the applied loads. These mechanisms, which are quite different to those that toughen metals for

\footnotetext{
${ }^{1}$ Materials Sciences Division, Lawrence Berkeley National Laboratory, Berkeley, California 94720, USA

${ }^{2}$ National Center for Electron Microscopy, Lawrence Berkeley National Laboratory, Berkeley, California 94720, USA

${ }^{3}$ Department of Materials Science and Engineering, University of California, Berkeley, California, 94720, USA

${ }^{*}$ To whom correspondence should be addressed. E-mail: roritchie@lbl.gov
} 
example, are created over so many dimensions (nano to macro) that it makes them very difficult to replicate in a synthetic material. From a fracture mechanics perspective, the presence of these mechanisms results in characteristic crack resistance-curve (R-curve) behavior where the fracture resistance actually increases with crack extension; in other words, these materials develop the majority of their toughening during crack growth, not during crack initiation. As a prime example, nacre consists of 95 vol.\% of layered aragonite $\left(\mathrm{CaCO}_{3}\right)$ platelets bonded by a thin layer of organic material, but yet exhibits a toughness (in energy terms) some three orders of magnitude higher than calcium carbonate (2). The hard aragonite provides for strength, but without a means to dissipate strain nacre would be brittle; however, large inelastic deformation generated by interlayer shearing through the organic phase allows for such strain redistribution (9) with the result that toughness is achieved through viscoplastic energy dissipation in the organic layer associated with the controlled, yet limited, sliding of the aragonite layers over each other. Although there is still some polemic regarding the mechanisms that restrain sliding, i.e., resistance from the lamellae nanoroughness (10), plastic deformation of the aragonite at the nano-level (11), the organic layer acting as a viscoelastic glue (12), or from the presence of mineral bridges $(2,13)$, the resulting toughness is remarkable. Attempts have been made to simulate this in synthetic materials, such as with lamellar materials fabricated with conventional processing, i.e., tape or slip casting; however, the resulting layer thicknesses are typically over two orders of magnitude larger than the $\sim 0.5 \mu \mathrm{m}$ aragonite platelet spacing in nacre $(14,15)$. It is true that such submicrometer layer spacings can be achieved by physical or chemical deposition, but not for bulk materials as the techniques are restricted to the fabrication of thin films (16-19).

Here we apply this natural concept of hierarchical design to ceramic/polymer $\left(\mathrm{Al}_{2} \mathrm{O}_{3} / \mathrm{PMMA}\right)$ hybrid materials which we are able to fabricate in bulk form by freeze casting (20-24). Using controlled freezing of ceramic-based suspensions in water, we are able to form large porous ceramic scaffolds (Fig. 1) with architectures that are templated by the ice crystals. We first employ directional freezing to promote the formation of lamellar ice with prescribed dimensions; this then acts as the "negative" for creation of the layered ceramic scaffolds which are subsequently infiltrated with the polymeric second phase. In addition to making lamellar structures, we can also fabricate nacre-like "brick-and-mortar" structures, with very high ceramic content, by subsequently pressing the scaffolds in the direction 
perpendicular to the lamellae in order to collapse them followed by a second sintering step to promote densification and the formation of ceramic bridges between the "bricks". Using such techniques, we have made complex hierarchical architectures where we can refine the lamellae thickness, control their macroscopic orientation, manipulate the chemistry and roughness of the inter-lamellae interfaces, and generate a given density of inorganic bridges, all over a range of size-scales.

In an attempt to replicate the microstructural design of nacre, we reduced the lamellae thickness to $5 \mu \mathrm{m}$ (this is still $\sim 10$ times thicker than the natural material) (Fig. 2A-B), and used sucrose as an additive to the ceramic slurries. Sucrose modifies the viscosity and phase diagram of the solvent resulting in the formation of ice crystals with a characteristic microscopic roughness and bridge density similar to that in nacre (Fig. 2C-D) (25). Control of the lamellae roughness provided a mechanical means to manipulate adhesion at the inorganic/organic layer interfaces. This can be complemented at the molecular level by chemical grafting a methacrylate group onto the ceramic surfaces prior to PMMA infiltration using in situ free radical polymerization; the presence of the methacrylate groups then acts to promote stronger covalent bonding between the two phases. To achieve a macroscopic alignment of the lamellae similar to nacre (over millimeter dimensions or more), we patterned the cold finger on which the ice nucleates using parallel, $\sim 40 \mu \mathrm{m}$, grooves. Brick-and-mortar architectures, prepared through pressing of the lamellar materials and subsequent sintering, have a much larger ceramic content (up to 80 vol.\%). The ceramic "bricks" are 5-10 $\mu \mathrm{m}$ wide and 20-100 $\mu \mathrm{m}$ long. While the polymer layers have an overall average thickness of $\sim 1-2 \mu \mathrm{m}$, there are large areas in which the alumina bricks are separated by sub-micrometer polymer films akin to the microstructure of natural nacre. After infiltrating these porous scaffolds with the polymer, we produced a series of $\mathrm{Al}_{2} \mathrm{O}_{3} / \mathrm{PMMA}$ hybrid composites (Fig 2B), with hierarchical structures spanning multiple length scales that exhibit distinctive structural and mechanistic features similar to those in nacre.

Matching the structural features of natural materials is not easy but attaining their unique combinations of mechanical properties is a much more difficult exercise and has in fact been rarely achieved. In natural and biological materials, desired properties are often achieved in a directional fashion $(2,26)$; indeed, certain engineering materials have also been 
optimized with highly anisotropic properties, as in multilayer ceramic armor materials to laminated epoxy/carbon fiber composites and directionally-solidified turbine blade alloys for aerospace engineering (27-29). Similarly, the flexural strengths of our ice-templated hybrid materials are high in the direction perpendicular to the lamellae and comparable to that of alumina, with values of 120 to $210 \mathrm{MPa}$ for the lamellae and brick-and-mortar structures, respectively (Fig. 3A). More importantly though, corresponding plane-strain $K_{\text {Ic }}$ fracture toughnesses (which represent values for crack initiation) are almost double of what could be expected from the simple "rule of mixtures" of $\mathrm{Al}_{2} \mathrm{O}_{3}$ and PMMA (Fig. 3B). Note that the reference alumina values here correspond to bulk samples prepared by slip casting in our laboratory. The suspensions for slip-casting and freeze casting use the same starting powders and similar solid contents with identical sintering cycles (30). Slip casting was selected because, as for freeze casting, the ceramic sample forms through the packing of powders from a liquid suspension.

Whereas grafting to improve $\mathrm{Al}_{2} \mathrm{O}_{3} / \mathrm{PMMA}$ interface adhesion (Fig. 3C-D) resulted in a mildly higher strength and initiation toughness for lamellar structures, a very significant increase was seen for the brick-and-mortar structure (Fig. 3A-B). However, the most striking feature of our synthetic composites is that they actually replicate the mechanical behavior of natural materials; specifically, they display large $(>1 \%)$ inelastic strains when loaded in tension (Fig. 4A), and develop exceptional toughness for crack growth (Fig. 4B). Like many hard mineralized bio-composites, the ice-templated materials exhibit a high degree of inelasticity, despite the brittle nature of their main ceramic constituent. While single-value linear-elastic parameters based on crack initiation, such as $K_{\mathrm{Ic}}$, have traditionally been used to quantify toughness, they cannot capture, nor even represent, the multiple length-scale toughening acting in these composites that lead to their characteristic R-curve behavior where the fracture resistance actually increases with crack extension. Human cortical bone, for example, derives more than $75 \%$ of its toughness during crack growth, which is not reflected in its reported $K_{\mathrm{Ic}}$ toughness value of 2 to $7 \mathrm{MPa} \sqrt{\mathrm{m}}$; the true toughness of bone (in the transverse orientation) is actually more than $20 \mathrm{MPa} \sqrt{\mathrm{m}}$ when the crack-growth toughening contributions are considered (26). To evaluate the toughness of these materials requires nonlinear elastic fracture mechanics to characterize the contributions from inelastic deformation and more importantly R-curve analysis to characterize the toughness associated 
with crack growth, both of which are afforded by the use of the $J$-R curve. Our brick-andmortar structures comprise $\sim 80 \%$ alumina yet display a remarkable $1.4 \%$ strain to failure. Correspondingly we used the $J$-R curve approach to fully define their toughness properties. Results (plotted for comparison in terms of a stress intensity $K_{\mathrm{J}}$ back-calculated from the $J$ measurements) are shown in Fig. 4B where it is apparent that both natural (nacre) and our synthetic composites exhibit extensive rising R-curve behavior indicative of extensive extrinsic toughening. The degree of toughening in our hybrid composites clearly surpasses that of nacre (the often cited "gold standard" in biomimetic design). Our $\mathrm{Al}_{2} \mathrm{O}_{3} / \mathrm{PMMA}$ lamellar and brick-and-mortar structures reach (steady-state) fracture toughnesses $K_{\mathrm{Jc}}$ of 15

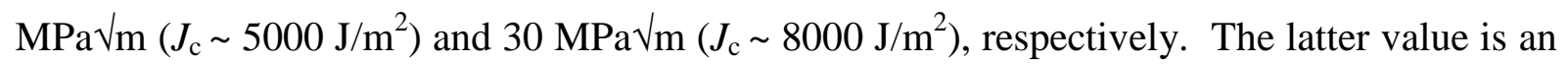
outstandingly high toughness for a material consisting of $80 \%$ alumina with a strength above $200 \mathrm{MPa}$; it is far in excess of the toughness of either constituent. Indeed, the toughnesses of our best hybrid materials are an order of magnitude higher than standard hot-pressed homogeneous nanocomposites consisting of $500 \mathrm{~nm} \mathrm{Al}_{2} \mathrm{O}_{3}$ particles dispersed in PMMA with the same nominal composition. Similar to the fine-grained $(1 \mu \mathrm{m})$ alumina, these homogeneous $\mathrm{Al}_{2} \mathrm{O}_{3} / \mathrm{PMMA}$ composites display minimal extrinsic toughening and consequently no rising R-curve behavior with characteristically very low fracture toughness below $2 \mathrm{MPa} \sqrt{\mathrm{m}}$ (Fig. 4B). We believe that result illustrates the importance of the notion of hierarchical design in promoting toughening mechanisms at multiple length scales as a path to create materials with unique combinations of strength and fracture resistance.

Mechanistically, we can identify the salient sources of toughening in these hybrid composites by performing additional fracture toughness tests in situ in the scanning electron microscope. This technique allows us the opportunity to quantitatively measure the R-curve while simultaneously monitoring the evolution of damage mechanisms ahead of the crack tip and extrinsic toughening mechanisms that result in the wake; furthermore, we can image in real time how these mechanisms are affected by the materials architecture (26). Our results show that there is clearly a confluence of toughening mechanisms acting at several lengthscales, as in biological materials. Most importantly, akin to nacre the microstructural damage is not at all localized but rather widely distributed over very large (millimeter-scale) dimensions (Fig. 4C). In general, in addition to inelastic deformation within the polymer, which can be seen in the form of micrometer-scale tearing in Fig. 5A, this damage principally 
takes the form of multiple microcracks contained within the ceramic layers. There are also elongated voids within the polymeric interlayers, many of which result from the processing, but which appear to promote local plastic flow and grow during loading. These brittle and ductile damage processes appear to be quite distinct with little evidence of interaction. The presence of such damage initiated ahead (and distinct from) the main growing crack leads to the formation of so-called "uncracked-ligament bridging" (31) in the wake of the crack tip. These elastic bridges (which are quite distinct from the pre-existing ceramic bridges between layers) are formed in situ before the main growing crack links to the microcracks and voids; the bridges effectively span the crack and carry load that would otherwise be used to promote macroscopic crack advance (32). Of note here is that this mechanism is one of the major origins of toughness in cortical bone and tooth dentin $(6,7)$, not to mention rocks and metalmatrix composites (31). In addition, the elongated voids in the polymeric layers (Fig. 4C) can act as very effective local crack arresters, as with the well known Cook-Gordon mechanism (33) in laminates; furthermore, the energy dissipation to break the resulting crack bridges leads to ductile-phase toughening (34), which is a common source of fracture resistance in polymer- and metal-matrix composites. Accordingly, through control of the structural architecture at several length-scales, we have managed to combine a multiple of toughening mechanisms operating over a large range of dimensions in order to generate extensive cracktip shielding and hence unprecedented fracture resistance for a ceramic-based material.

The difference in the lamellar vs. the brick-and-mortar structures lies in the precise nature and relative contribution of these toughening mechanisms. In the lamellar composites, chemical grafting of the organic-inorganic interfaces does not appear to have too much of an effect. However, it is observed that the weaker $\mathrm{Al}_{2} \mathrm{O}_{3} / \mathrm{PMMA}$ (non-grafted) interfaces promote crack deflection and "delamination" along the interface (Fig. 3C-D). This facilitates the formation of uncracked-ligament bridging (as described above) but also frictional sliding ("sliding interference") along the rough debonded interface with consequent energy dissipation associated with deformation within the soft phase. The situation is different with the brick-and-mortar structures where the highest fracture toughness of over $30 \mathrm{MPa} \sqrt{\mathrm{m}}$ is achieved with high strength, grafted interfaces. As with the lamellar materials, the brick-andmortar microstructures exhibit extensive elastic bridging; however, unlike the lamellar materials there is no fracture of the ceramic bricks, rather there is some degree of "pull-out" 
between the bricks that in some respects replicates at microscopic dimensions the nanoscale phenomena observed in nacre (Fig. 5) (35). This form of toughening is particularly potent and the prime source of the fracture resistance of most monolithic structural ceramics with engineered grain boundaries, i.e., course-grained $\mathrm{Al}_{2} \mathrm{O}_{3}, \mathrm{Si}_{3} \mathrm{~N}_{4}$ and $\mathrm{SiC}$ (36-43). However, the contribution from bridging alone does not account for the fact that the observed toughness of the best brick-and-mortar $80 \%$ alumina structure $\left(J_{c} \sim 8000 \mathrm{~J} / \mathrm{m}^{2}\right)$ is over 300 times higher in terms of energy than the toughness of its main constituent, $\mathrm{Al}_{2} \mathrm{O}_{3}\left(J_{\mathrm{c}} \sim 26 \mathrm{~J} / \mathrm{m}^{2}\right)$. We believe that of the various hybrid materials that we have fabricated, this structure best mimics nacre. Freeze casting followed by pressing and a second sintering stage results in a microstructures with high ceramic contents characterized by the sub-micrometer lubricating polymer interlayers between ceramic "bricks" (deformation and micrometer-scale tearing in these interlayers can be seen in Fig. 5A); this combined with the roughness of the ceramic interfaces and the presence of stiff ceramic bridges between grains with micrometer and submicrometer dimensions (Figs. 2 and 4), promotes controlled sliding and "sliding interference" (Fig. 5) between the rough ceramic interlayers, thereby enhancing the toughness through extremely efficient energy dissipation. The result is synthetic materials which like nacre and bone are far tougher that what could be expected from the simple mixture of their constituents (Fig 6A).

A better appreciation of the unique mechanical properties of these freeze-cast composites can be gained by comparing them to other materials. By combining two relatively ordinary phases, i.e., a hard yet brittle ceramic with a relatively soft (in comparison) polymer, we have synthesized primarily (ceramic) alumina hybrid structures with specific strength and toughness properties that in the favorable directions match those of engineering (metallic) aluminum alloys (Fig. 6B) and moreover display a higher stiffness. This has been possible through the development of hierarchical architectures that combine toughening mechanisms acting at multiple scales, from sub-micrometer dimensions (i.e., the ceramic bridges between lamellae or bricks or the inelastic polymer deformation) and higher. These results highlight the tremendous potential of the biomimetic approach, and suggest promising strategies for structural optimization. In particular, a key attribute of nacre that has been extremely difficult to replicate in practical dimensions is the fact that the structure consists of $95 \mathrm{vol} \%$ ceramic with very little of the organic soft phase which is actually distributed as a thin (2-3 nm) 
protein film that acts like a lubricant. At present, our materials contain too much of the soft phase, and our ceramic layer thicknesses are still somewhat coarse in comparison to nacre; indeed, a reduction in the polymer content and refinement of the ceramic layers will almost certainly improve strength and provide additional nanoscale toughening mechanisms akin to those acting in natural materials. In this regard, our current studies are focused on the development of these hybrid structures with much higher inorganic content, the manipulation of the properties of the soft lubricating phase, and extending this concept to other material combinations, principally metal-infiltrated ceramics.

\section{References and Notes}

1. G. Mayer, Science 310, 1144 (Nov 18, 2005).

2. M. A. Meyers, P. Y. Chen, A. Y. M. Lin, Y. Seki, Progress in Materials Science 53, 1 (Jan, 2008).

3. C. Ortiz, M. C. Boyce, Science 319, 1053 (Feb 22, 2008).

4. J. Aizenberg et al., Science 309, 275 (Jul 8, 2005).

5. F. Barthelat, H. D. Espinosa, Experimental Mechanics 47, 311 (Jun, 2007).

6. R. K. Nalla, J. H. Kinney, R. O. Ritchie, Biomaterials 24, 3955 (Oct, 2003).

7. R. K. Nalla, J. J. Kruzic, J. H. Kinney, R. O. Ritchie, Biomaterials 26, 217 (Jan, 2005).

8. R. O. Ritchie, Materials Science and Engineering A 103, 15 (Aug, 1988).

9. R. Z. Wang, Z. Suo, A. G. Evans, N. Yao, I. A. Aksay, Journal of Materials Research 16, 2485 (Sep, 2001).

10. A. G. Evans et al., Journal of Materials Research 16, 2475 (Sep, 2001).

11. X. D. Li, W. C. Chang, Y. J. Chao, R. Z. Wang, M. Chang, Nano Letters 4, 613 (Apr, 2004).

12. B. L. Smith et al., Nature 399, 761 (Jun 24, 1999).

13. F. Song, A. K. Soh, Y. L. Bai, Biomaterials 24, 3623 (Sep, 2003).

14. H. M. Chan, Annual Review of Materials Science 27, 249 (1997).

15. J. S. Moya, Advanced Materials 7, 185 (Feb, 1995).

16. A. Sellinger et al., Nature 394, 256 (Jul 16, 1998).

17. Z. Y. Tang, N. A. Kotov, S. Magonov, B. Ozturk, Nature Materials 2, 413 (Jun, 2003).

18. L. J. Bonderer, A. R. Studart, L. J. Gauckler, Science 319, 1069 (Feb 22, 2008).

19. P. Podsiadlo et al., Science 318, 80 (Oct 5, 2007).

20. S. Deville, E. Saiz, R. K. Nalla, A. P. Tomsia, Science 311, 515 (Jan 27, 2006).

21. S. Deville, E. Saiz, A. P. Tomsia, Acta Materialia 55, 1965 (Apr, 2007).

22. T. Fukasawa, M. Ando, T. Ohji, S. Kanzaki, Journal of the American Ceramic Society 84, 230 (Jan, 2001).

23. T. Fukasawa, Z. Y. Deng, M. Ando, T. Ohji, Y. Goto, Journal of Materials Science 36, 2523 (2001).

24. K. Araki, J. W. Halloran, Journal of the American Ceramic Society 87, 1859 (Oct, 2004). 
25. S. Deville, E. Munch, E. Saiz, A. P. Tomsia, unpublished results (2008).

26. K. J. Koester, J. W. Ager, R. O. Ritchie, Nature Materials 7, 672 (Aug, 2008).

27. A. Tasdemirci, I. W. Hall, B. A. Gama, M. Guiden, Journal of Composite Materials 38, 995 (2004).

28. D. R. Johnson, X. F. Chen, B. F. Oliver, R. D. Noebe, J. D. Whittenberger, Intermetallics 3, 99 (1995).

29. R. M. Jones, Mechanics of composite materials (Taylor \& Francis, Philadelphia, PA, ed. $\left.2^{\text {nd }}, 1999\right)$, pp. xvi, 519 p.

30. Supporting online material.

31. J. K. Shang, R. O. Ritchie, Metallurgical Transactions A 20, 897 (May, 1989).

32. Instead of crack extension being solely associated with the main crack tip growing forward, crack advance also can occur by microcracks (or other damage) initiated ahead of the main crack tip linking back to the tip.

33. J. Cook, C. C. Evans, J. E. Gordon, D. M. Marsh, Proceedings of the Royal Society of London Series A 282, 508 (1964).

34. L. S. Sigl, P. A. Mataga, B. J. Dalgleish, R. M. McMeeking, A. G. Evans, Acta Metallurgica 36, 945 (Apr, 1988).

35. A. Y. M. Lin, P. Y. Chen, M. A. Meyers, Acta Biomaterialia 4, 131 (Jan, 2008).

36. P. F. Becher et al., Journal of the American Ceramic Society 81, 2821 (Nov, 1998).

37. J. J. Cao, W. J. MoberlyChan, L. C. DeJonghe, C. J. Gilbert, R. O. Ritchie, Journal of the American Ceramic Society 79, 461 (Feb, 1996).

38. J. J. Kruzic, R. M. Cannon, R. O. Ritchie, Journal of the American Ceramic Society 87, 93 (Jan, 2004).

39. J. J. Kruzic, R. M. Cannon, R. O. Ritchie, Journal of the American Ceramic Society 88, 2236 (Aug, 2005).

40. F. F. Lange, Journal of the American Ceramic Society 56, 518 (1973).

41. Y. W. Mai, B. R. Lawn, Journal of the American Ceramic Society 70, 289 (Apr, 1987).

42. N. P. Padture, B. R. Lawn, Journal of the American Ceramic Society 77, 2518 (Oct, 1994).

43. P. L. Swanson, C. J. Fairbanks, B. R. Lawn, Y. W. Mai, B. J. Hockey, Journal of the American Ceramic Society 70, 279 (Apr, 1987).

44. U. G. K. Wegst, M. F. Ashby, Philosophical Magazine 84, 2167 (Jul 21, 2004).

45. This work was supported by the Director, Office of Science, Office of Basic Energy Sciences, Division of Materials Sciences and Engineering, of the U.S. Department of Energy under Contract No. DE-AC02-05CH11231 


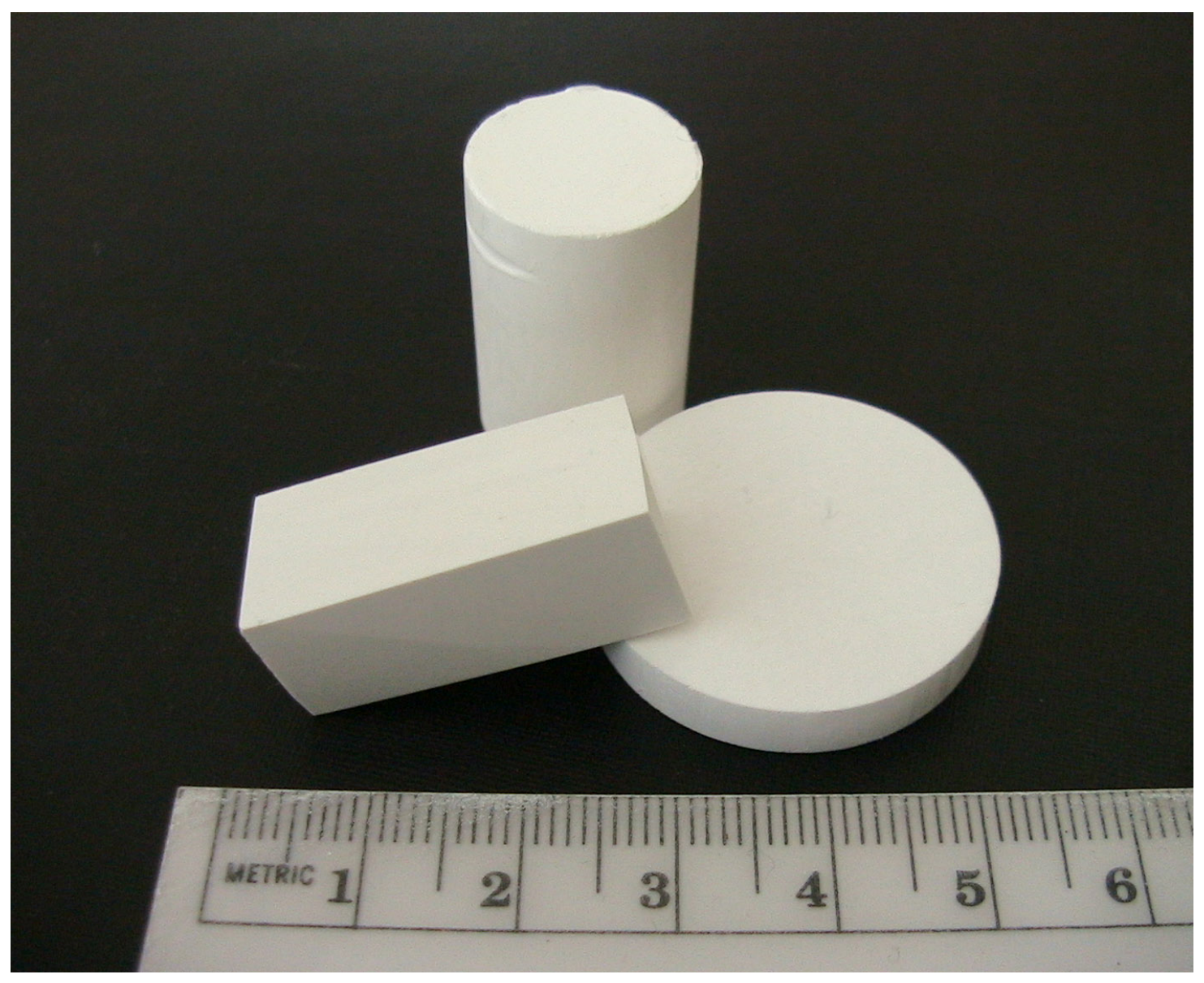

Figure 1. Fabrication of bulk hybrid composite materials. Porous scaffolds of practical dimensions obtained by freeze casting of ceramic suspensions. The control of the processing conditions leads to macroscopic samples that exhibit lamellar structures oriented over several centimeters. 

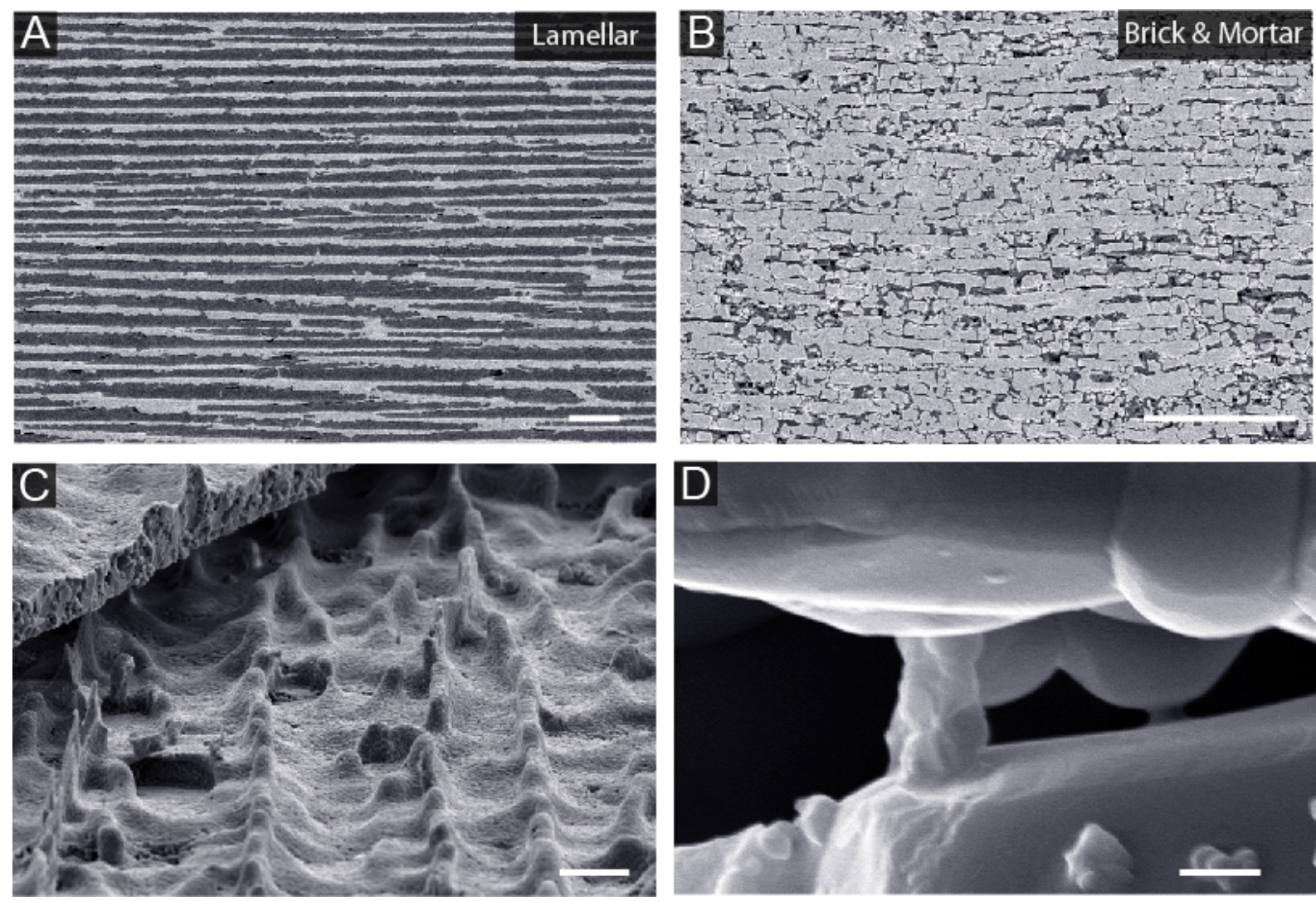

Figure 2. Structure of ice-templated materials. (A) $\mathrm{Al}_{2} \mathrm{O}_{3} / \mathrm{PMMA}$ lamellar composites have been fabricated using freeze casting of ceramic suspensions followed by polymer infiltration (the lighter phase is the ceramic; the dark phase is the polymer). (B) Brick-and-mortar architectures are prepared through pressing of the lamellar materials and subsequent sintering and have much larger ceramic contents (up to $80 \mathrm{vol} . \%$ ). (C) Using sucrose as an additive to the freeze casting slurry the growing ice crystals develop a characteristic surface topography that translates into a microscopic roughness in the ceramic walls. (D) The process also results in the formation of ceramic bridges between lamellae (due to the trapping of ceramic particles by the growing ice) or bricks (the bridges form during the second sintering steps). By limiting sliding they provide very effective toughening mechanisms in natural and synthetic materials. The alumina grain size inside the lamellae or bricks is of the order of $1 \mu \mathrm{m}$. The bars are $100 \mu \mathrm{m}$ in (A) and (B) $10 \mu \mathrm{m}$ in (C) and $600 \mathrm{~nm}$ in (D). 

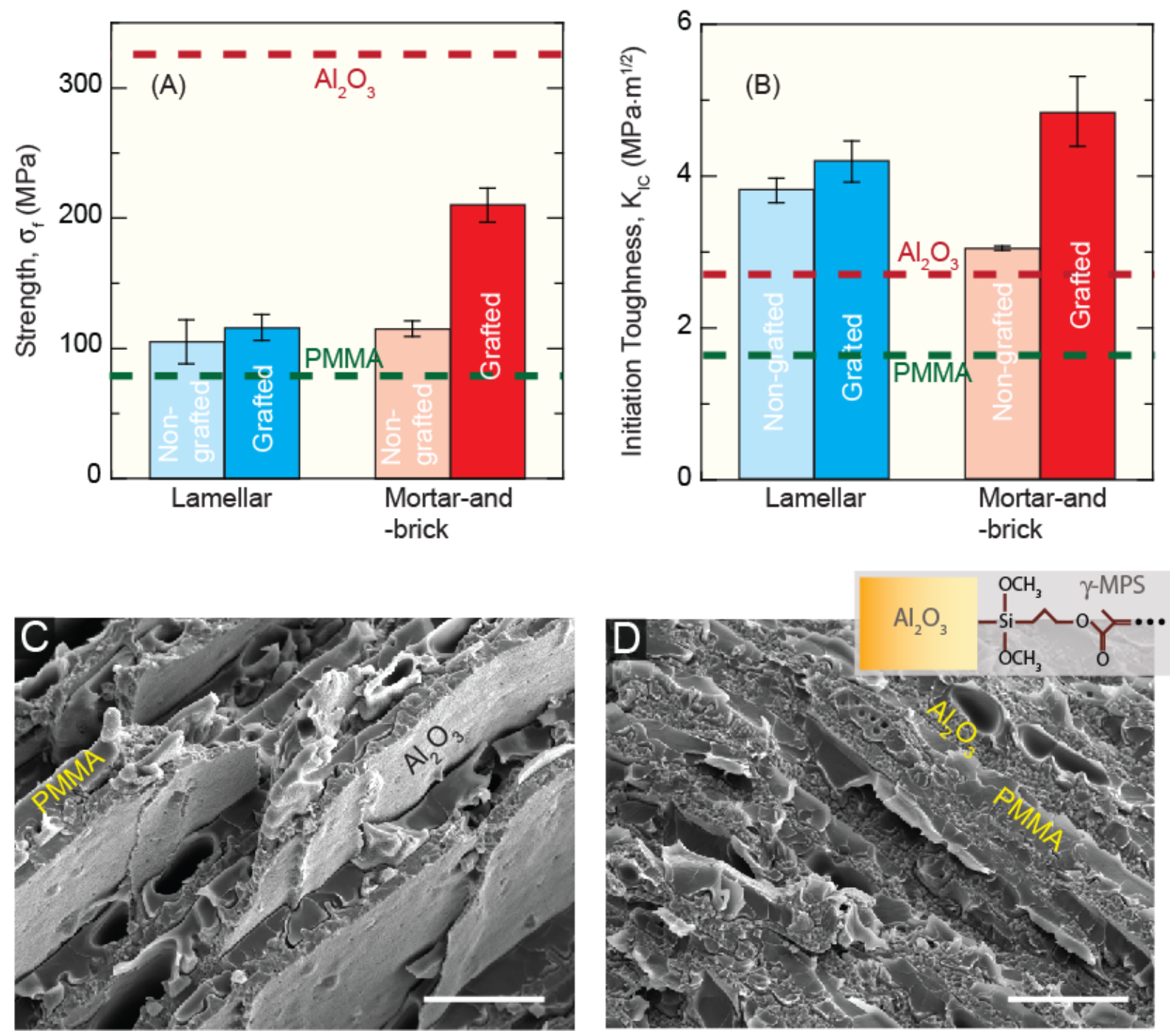

Figure 3. Influence of interfacial chemistry on the mechanical response. (A) The strength of the hybrid composites can reach values above $200 \mathrm{MPa}$. (B) The crack-initiation fracture toughness, $K_{I C}$, can be up to twice that of the materials components. Chemical grafting increases the adhesion at the organic/inorganic interface and enhances both strength and initiation toughness. The error bars represent $+/-$ the standard deviation. (C) The scanning electron micrograph of the fracture surface of a lamellar material with non-grafted interfaces (which leads to weaker boundaries) shows extensive interface delamination during fracture. (D) Chemical grafting (see insert) result in better adhesion (stronger boundaries) and fracture surfaces that are comparatively much flatter. 

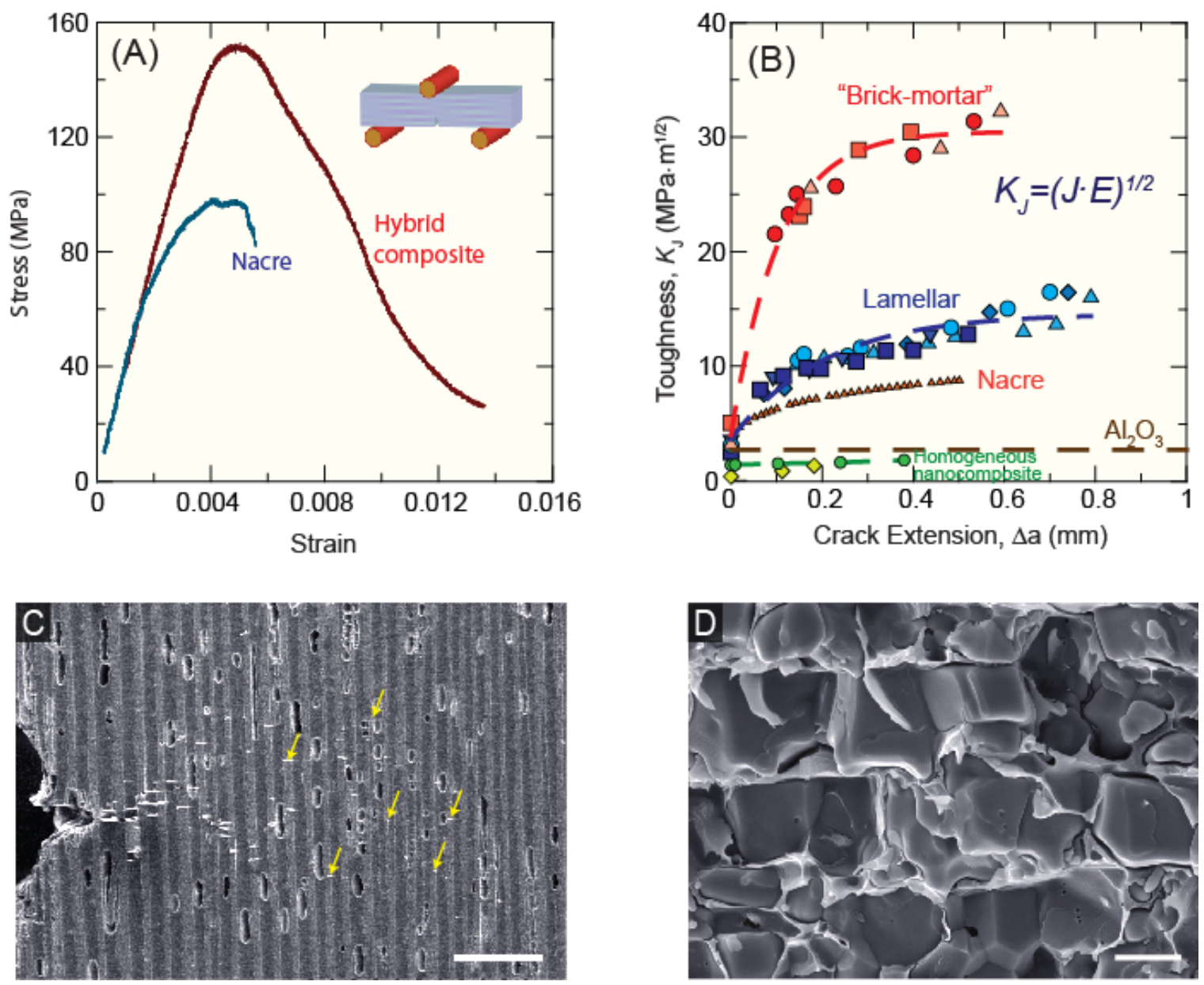

Figure 4. Mechanical response and toughening mechanisms in the synthetic hybrid composites.

(A) Bending stress-strain curves for the $\mathrm{Al}_{2} \mathrm{O}_{3}$ /PMMA hybrid materials mimic those of nacre, and show $>1 \%$ inelastic deformation prior to failure. The curves correspond to non-grafted lamellar hybrid composite and hydrated nacre (abalone shell). (B) These materials show exceptional toughness for crack growth, akin to natural composites, and display significant rising resistance-curve behavior. Almost negligible toughening is observed in nanocomposites consisting of $500 \mathrm{~nm} \mathrm{Al}_{2} \mathrm{O}_{3}$ particles dispersed in PMMA. (C) Scanning electron micrograph taken during an in situ R-curve measurement of a lamellar structure. The image taken during crack propagation shows two of the toughening mechanisms acting at large scales: the wide distribution of damage (over millimeter dimensions) in the form of contained microcracking within the ceramic layers (yellow arrows point to some of these microcracks) and the voids in the polymer layers. (D) Fracture surface of a grafted brick-and-mortar structure: controlled sliding contributes to a rise of the crack-growth toughness to values that can be over 300 times higher (in energy terms) than that of $\mathrm{Al}_{2} \mathrm{O}_{3}$. In addition to the roughness of the ceramic surfaces and the inorganic bridges between ceramic bricks, a principal reason for the controlled sliding is the presence of a sub-micrometer polymer film between alumina blocks. The bars are $250 \mu \mathrm{m}$ in (C) and $3 \mu \mathrm{m}$ in (D). 

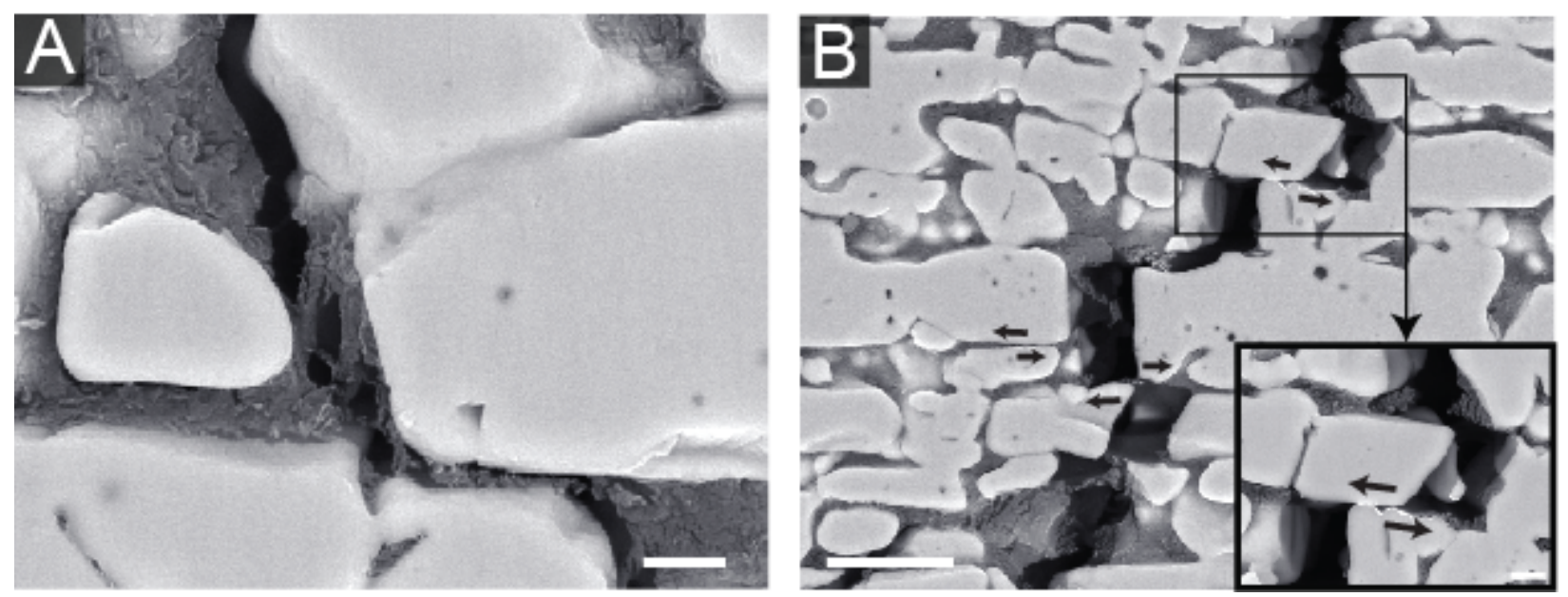

Figure 5. Toughening mechanisms in brick-and-mortar microstructures. In situ imaging of crack propagation in brick-and-mortar structures shows clear evidence of (A) polymer tearing and stretching over micrometer dimensions (as has also been observed in the organic phase of nacre), and (B) "pull out" and frictional sliding between ceramic bricks (see also insert). The thin bright lines between the sliding grains in the inset indicate electrical charging in the SEM resulting from the deformation of the gold coating during sliding. The bars are $2 \mu \mathrm{m}$ in (A) and the insert in (B) and $10 \mu \mathrm{m}$ in (B). 

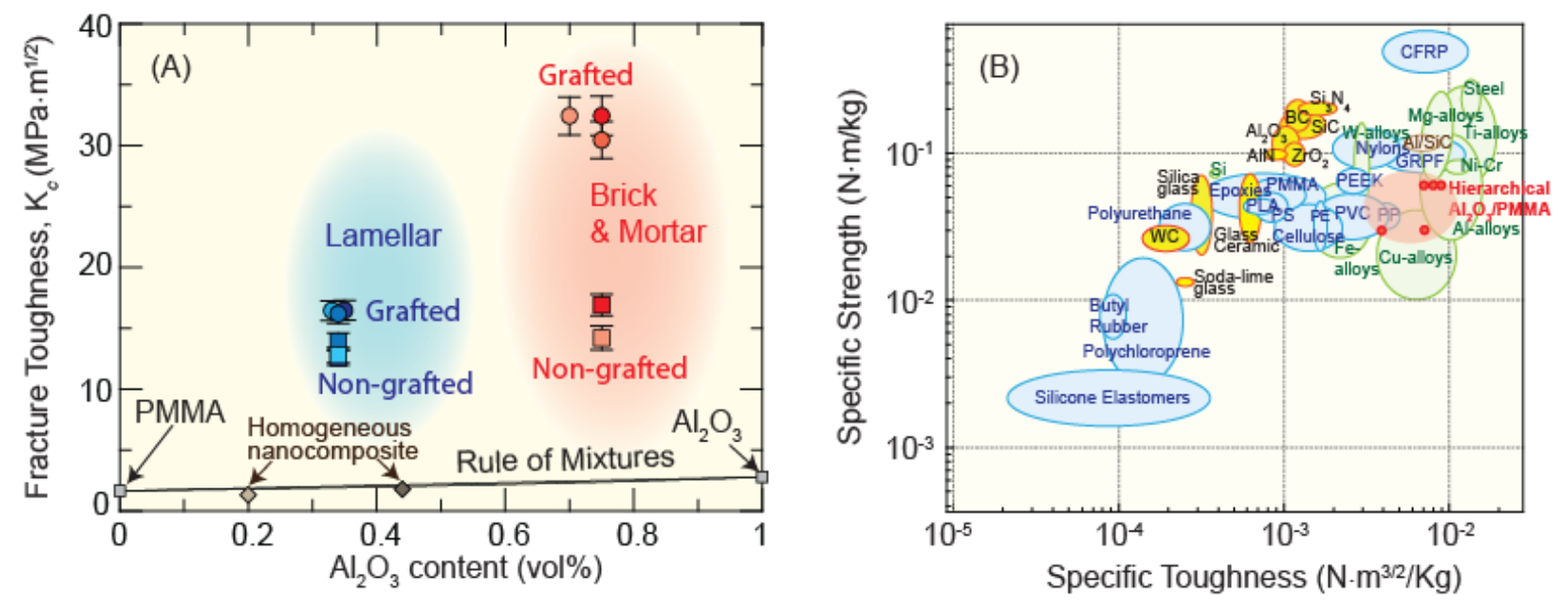

Figure 6. Specific Strength/Toughness Properties of Hybrid Composites Comparable to Aluminum

Alloys. (A) Fracture toughness for lamellar and brick-and-mortar composites. The toughness of the pure components, along with that from the rule of mixtures, is also shown. It is apparent that both types of composites are significantly tougher than their constituents. In particular, the brick-and-mortar composites display $K_{\mathrm{c}}$ fracture toughnesses over an order of magnitude larger than their principal constituent phase $\mathrm{Al}_{2} \mathrm{O}_{3}$. (The circles correspond to grafted (strong) interfaces and the squares to nongrafted (weaker) interfaces. The error bars represent $+/-$ the standard deviation). (B) Specific strength and toughness (i.e., properties normalized by density) for a wide range of materials systems (44), showing that our hierarchical $\mathrm{Al}_{2} \mathrm{O}_{3}$ /PMMA composites, some containing $~ 80$ vol.\% alumina ceramic, have strength/toughness properties comparable with that of metallic aluminum alloys. 